\title{
SECUENCIAS DE CONECTORES EN EL CORPUS VAL.ES.CO.
}

\section{SEQUENCES OF CONNECTIVES IN THE VAL.ES.CO CORPUS}

\section{Maria Josep Cuenca Ordinyana}

Universitat de València

Resumen

En este artículo, analizamos las secuencias de conectores que aparecen en conversaciones coloquiales en español del corpus Val.Es.Co. Siguiendo la propuesta presentada en Cuenca y Marín (2009) y revisada y aplicada al inglés en Cuenca y Crible (2019), diferenciamos tres tipos de relaciones entre dos o más conectores consecutivos: yuxtaposición, adición y composición. El análisis permite describir y ejemplificar cada tipo de secuencia, identificar las secuencias que incluye el corpus de análisis y estudiar con un poco más de detalle los casos de conectores o marcadores compuestos.

PALABRAS CLAVE: conexión, conectores, secuencias de conectores, conversación coloquial.
Abstract

In this paper, I analyze sequences of connectives in Spanish informal conversations from the Val.Es.Co corpus. Following the proposal presented in Cuenca y Marín (2009) and revisited and applied to English in Cuenca y Crible (2019), three degrees of integration of two or more consecutive connectives are proposed, namely, juxtaposition, addition and composition. The analysis allows us to describe and exemplify each type of sequence, identify the specific tokens found in the corpus of analysis and account for the case of compound connectives in more detail.

KEY WORDS: connection, connectives, sequence of connectives, informal conversation 


\section{INTRODUCCIÓN}

Hasta hace no mucho tiempo la concurrencia de conectores o marcadores del discurso era un tema que aparecía aquí y allá, de manera poco sistemática y a veces anecdótica, entre los numerosísimos trabajos dedicados al tema. Recientemente, este fenómeno parece suscitar un interés cada vez mayor, lo cual no deja de tener relación, como ya ha sucedido en otros casos, con que ha sido 'descubierto' por investigadores que trabajan sobre el inglés. Esto nos augura un buen y fructífero futuro para un aspecto al que algunos investigadores del ámbito hispánico ya hemos hecho aportaciones importantes y sistemáticas desde hace años (cf., por ejemplo, González, 2001, 2004; Pons, 2008, Cuenca y Marín, 2009).

En este trabajo presentamos un análisis de las secuencias de conectores en cuatro conversaciones (una por década desde los años 80 ) del corpus Val.Es.Co: la conversación 1 (1989, 2 hablantes), la 14 (1994, 4 hablantes), la 15 (2007, 4 hablantes) y la 42 (2011, 3 hablantes). Estas conversaciones suman un total de 22.204 palabras y tienen una duración aproximada de 2 horas.

El objetivo general de esta investigación es aplicar la clasificación de las secuencias de marcadores del discurso que establecimos en 2009 (Cuenca y Marín, 2009) y que hemos revisado recientemente (Cuenca y Crible, 2019) a un corpus de conversaciones informales en español. Proponemos diferenciar tres tipos de relaciones entre conectores consecutivos: yuxtaposición, adición y composición. El análisis permitirá describir y ejemplificar cada tipo de secuencia, identificar las secuencias que incluye el subcorpus y analizar con un poco más de detalle los casos de conectores o marcadores compuestos.

La propuesta clasificatoria presentada aquí se ha aplicado anteriormente al Corpus Audiovisual Plurilingüe de la UB, en concreto a narraciones orales obtenidas a través de entrevistas semidirigidas en catalán y en español (Cuenca y Marín, 2009), y al corpus DisFrEn, que contiene textos orales en francés (Crible, 2018) y en inglés (Crible, 2017, 2018; Cuenca y Crible, 2019). La aplicación al corpus Val.Es.Co, aunque limitada en cuanto a la extensión del corpus de análisis, abre la puerta a un estudio más exhaustivo y a una futura comparación con otras lenguas, como se apunta en las conclusiones.

\section{SECUENCIAS DE CONECTORES}

Hablamos de secuencias o concurrencia de conectores cuando dos o más conectores tienen alcance parcial o total sobre una unidad discursiva $(Q)$ y la relacionan con una unidad anterior $(P)$, es decir: " $P c_{1} c_{2}\left(c_{n}\right) Q$ ". En general, se trata de secuencias de dos conectores (1), pero se pueden encontrar secuencias mayores, de tres (2) e incluso más de tres conectores (3).

(1) A: [iella ha acabao] la carrera y ha hecho un máster ya y todo!

C: ¿de qué tienes tú el máster? §

B: §bueno pero todavía me faltaa el trabajo final // el másteer en estudios hispánicos $§(42,54)$

(2) A: [síi $\downarrow] / /$ te dan un guion $\uparrow$ más o menos para que túu rellenes $\uparrow \mathbf{y}$ entonces pues tú puedes poner $\uparrow /$ todo lo que quieras/ desde donde está ubicado el colegio $\uparrow /$ los profesores que te han tocaado $\uparrow /$ tus experiencias personales $\uparrow /$ si te ha gustado o no te ha gustado $\uparrow(1,57)$ 
(3) A: Alicante Valencia y Castellón/ yy- y claro $\downarrow /$ y luego aparte $\uparrow /$ como a lo mejor pues de Ciencias o dee Humanas// o Filología $\uparrow$ no [se ha $(())](1,27)$

En (1) encontramos dos conectores, bueno y pero, iniciando una respuesta con la que el hablante $B$ niega la presuposición de $C$ (que ha acabado ya el máster, como la persona de la que se habla antes) mediante un desacuerdo parcial o atenuado seguido de una marca de contraste; en (2) son tres los conectores ( $y$ entonces pues) que marcan una frontera en una explicación e introducen un enunciado que continúa la explicación dentro de un mismo turno; en (3), encontramos cuatro conectores consecutivos, y luego aparte como, con diferentes grados de integración y funciones más o menos separadas: y luego aparte marcan una frontera o cambio de foco de atención en el discurso e indican continuidad, es decir, tienen una función estructural (dominio textual), mientras que como introduce una causal (dominio ideacional o proposicional).

En la línea planteada en Cuenca y Crible (2019), excluimos del concepto de concurrencia unidades lexicalizadas, como o sea que, contactos no estructurales causados por repeticiones, vacilaciones o reinicios y conectores separados por largas pausas, como se muestra en los ejemplos siguientes. ${ }^{1}$

(4) A: [tienes que] captarles la atención $\downarrow$ y como cada niño $\uparrow / /$ tienes que darle un poco a cada niño loo- lo suyo $\downarrow$ entonces $\uparrow / / \mathbf{y}$ ¡claro! es muy difícil hoy en día eso $\downarrow(1,177)$

(5) A: § para que me oigan $\uparrow$ o / yo a la mía ¿no?/ entonces $\uparrow$ nada// bueno $\rightarrow /$ he ido $\uparrow$ creo que dos veces a la discoteca ¿no?/ yy-// peroo- que te quiero decir/ NO me hace mucho $\rightarrow /$ y $\uparrow$-y prefiero $\uparrow$ pues yo qué sé/ cosas en plan verbena $\rightarrow \S(1,383)$

La concurrencia de conectores (o de marcadores discursivos o pragmáticos en general) no es un hecho marginal. Contamos ya con distintos estudios que ponen de manifiesto su relevancia cuantitativa en textos orales. ${ }^{2}$ Cuenca y Marín (2009) identifican un $16,48 \%$ de casos (149 de un total de 904 marcadores en un corpus de 5.913 palabras) en el subcorpus en catalán y un 17,25\% de casos (156 de 905 marcadores en un corpus de 5.929 palabras) en el subcorpus en español. Crible (2017), que adopta una perspectiva más amplia sobre el concepto de marcador del discurso, identifica un $15,77 \%$ de casos (670 de 4249 apariciones de marcadores) en inglés y un $23,85 \%$ de casos de concurrencias (1072 de 4494 apariciones de marcadores) en francés. Partiendo de una visión más restrictiva de los marcadores, Cuenca y Crible (2019) identifican 109 casos de un total de 34.434 palabras en un subcorpus del corpus de Crible (2017) en inglés.

Ciertamente, las cifras son relativas, puesto que dependen del corpus, del subgénero, de cada texto concreto y a veces incluso del hablante, pero presentan unos datos que

\footnotetext{
${ }^{1}$ Exclusiones similares pueden encontrarse en otros trabajos, como el de Lohmann y Koops (2016). Por otro lado, cabe notar que el concepto de marcador del discurso es diferente según los autores. En general, se incluyen elementos de carácter modal como ciertas interjecciones que típicamente introducen turno (como oh en inglés) o elementos como you know o claro. Por nuestra parte, siguiendo los principios planteados en Cuenca (2013), nos ceñimos a las cadenas de conectores y excluimos los contactos que incluyen marcas modales como ¿no?, claro o es que, muy frecuentes en la conversación coloquial, así como los casos de contacto con marcadores de cierre discursivo, como y nada, y eso, pero nada, pues nada. ${ }^{2}$ Se trata de un fenómeno no exclusivo de la oralidad. De hecho, el concepto de conector parentético, que hemos estudiado ampliamente en otros trabajos (cfr., p. ej., Cuenca, 2001, 2006, 2013), deriva de la combinación de conectores y conjunciones, como en el caso de y, en cambio o pero, de todos modos, propia del texto escrito y planificado. Ciertamente, las formas y las frecuencias cambian según el canal, tema que cabe explorar ulteriormente.
} 
indican que la secuencia de conectores no es casual, sino que responde a estrategias comunicativas que merece la pena estudiar.

La muestra del corpus Val.Es.Co que analizamos aquí contiene 168 secuencias de conectores en un total de 22.204 palabras, que representan 84 combinaciones distintas. Las secuencias más frecuentes (con 4 o más ocurrencias) son las que se muestran en (6):

(6) y entonces (21 ocurrencias), y luego (18), pero si (7), pero vamos (7), pero que (6), pero como (5), y que (5), y bueno (5), bueno pero (4), bueno pues (4), que si (4), y además (4).

Observamos que el primer elemento de una secuencia suele ser una conjunción básica ( $\mathrm{y}$, pero y menos frecuentemente que o porque) o el conector pragmático bueno. $\mathrm{El}$ segundo elemento suele ser un conector parentético (entonces, luego, además), un conector pragmático (vamos, bueno) o una conjunción subordinante (si, que, como, pues). En concreto:

a) En 140 de los 168 casos (83,3\%) el primer elemento es una conjunción, por orden de frecuencia: y (68 casos), pero (37), que (13), pues (13), porque (9). En 17 casos de los 28 restantes el primer elemento es bueno $(60,7 \%$ de otros conectores y $10,1 \%$ del total).

b) En cuanto al segundo elemento, los más frecuentes son los conectores parentéticos entonces (24 casos), luego (19) y además (9) y los conectores pragmáticos bueno (13) y vamos (a ver) (11). Tienen una cierta frecuencia algunas conjunciones (si (18), que (14), como (9)), pero algunos de sus usos no son propiamente conectivos, sino de carácter enunciativo o enfático. ${ }^{3}$

\section{SECUENCIAS DE MARCADORES DEL DISCURSO: BREVE ESTADO DE LA CUESTIÓN}

Las secuencias de marcadores discursivos se han estudiado teniendo en cuenta aspectos diversos y complementarios. Las principales aportaciones de carácter global se han centrado en la clasificación según el grado de integración de los marcadores, el análisis del orden de sus elementos (linearización) y la relación con la gramaticalización. ${ }^{4}$

\footnotetext{
${ }^{3}$ Diferenciamos tres tipos de conectores: las conjunciones (e.g. y, pero), los conectores parentéticos (e.g., sin embargo, además) y los conectores pragmáticos (e.g., bueno, mira). Los segundos se combinan con las conjunciones y suelen utilizarse, solos o en combinación, en el nivel textual, como introductores de unidades iguales o superiores a la oración o el enunciado independiente; en el caso de los parentéticos tienen un significado más específico y próximo a los valores proposicionales de adición, disyunción, contraste o consecuencia; los pragmáticos tienen un significado más procedimental y dependiente del contexto, vinculado a los dominios textual e interactivo, es decir, a las funciones estructurales y modales. Para una explicación más detallada de la propuesta categorial, véase Cuenca $(2006,2013)$.

${ }^{4}$ Existen otros trabajos centrados en grupos de marcadores, como los de Fraser (2013), que propone un análisis general de secuencias que incluyen dos marcadores contrastivos en inglés, como but, on the other hand o but instead, y extiende el análisis posteriormente Fraser (2015) a marcadores de consecuencia. Tanghe (2018) se ocupa de combinaciones que incluyen marcadores derivados de verbos de movimiento (anda, vamos, vaya y venga).
} 
En cuanto al grado de integración, son diversas las aportaciones, más o menos ocasionales o exhaustivas, según el caso. Basándose en el alcance del marcador, Luscher (1993) diferencia la adición de marcadores, cuando tienen diferente alcance sintáctico y semántico, de la composición, cuando los marcadores tienen igual alcance; en el segundo caso, considera que se trata de marcadores similares semánticamente con diferente grado de especificidad, como en el caso de mais pourtant, en francés. Desde una perspectiva más semántica y funcional, Hansen (1998) diferencia los conectores sumativos, que mantienen su significado individual (p. ej. ah bon), de los combinados, que constituyen un nuevo significado complejo (p. ej. eh bien).

González (2001, 2004), siguiendo la propuesta de Redeker (1991), parte de la distinción entre marcadores de estructura ideacional (o conectores) y marcadores de estructura pragmática. Los conectores son "semánticamente ricos", es decir, tienen una función gramatical y referencial, mientras que los marcadores pragmáticos son procedimentales, establecen relaciones de coherencia pragmática y facilitan la transmisión de la información al oyente; entre los marcadores pragmáticos diferencia los simples y compuestos. En un corpus de relato oral en inglés, identifica los siguientes marcadores compuestos: then well, okay anyhow, so anyway, so you know, well I mean y well so; en catalán, identifica muchas más formas, como bueno doncs, bueno clar, bueno aleshores/llavors, bé doncs, bé llavors/llavòrens, llavors bueno/bé/pues, aleshores bueno/doncs/clar, doncs mira/res. En este caso, todas las secuencias de elementos conectivos ( $\mathrm{y}$ alguno modal) que no corresponden a una conjunción se analizan como una secuencia, sin establecer diferencias en el grado de integración.

Por su lado, Pons (2008) analiza las secuencias que incluyen bueno y concluye que hay casos de adyacencia y casos de combinación según el alcance de los marcadores (diferente o igual). En una obra posterior (Pons, 2018) propone distinguir tres relaciones entre marcadores consecutivos: adyacencia (cuando los marcadores no están relacionados y se relacionan con unidades diferentes), combinación (cuando están relacionados, p. ej. y luego, y entonces; bueno pero, bueno pues) y lexicalización (cuando se convierten en rutinas conversacionales, p. ej. pues bueno).

En Cuenca y Marín (2009) se establecen tres grados de integración: yuxtaposición, adición y composición de marcadores del discurso. Estos tres grados se relacionan con patrones gramaticales, posición y alcance, y dominio conceptual o función más frecuentes. Así, la yuxtaposición prototípica (p. ej. y mientras) implica la secuencia de dos conjunciones que funcionan dentro de la oración o acto y se interpretan en el dominio ideacional o proposicional. La adición prototípica (p. ej. pero, en cambio) implica una conjunción seguida de un conector parentético o pragmático que se sitúa dentro de un acto o turno y que suele marcar transiciones discursivas menores; el dominio puede ser el ideacional o el textual, pues suelen expresar significados proposicionales (como el contraste) o estructurales (como el cambio de tema o subtema). La composición prototípica (p. ej. pues vale) incluye combinaciones de conectores parentéticos y/o pragmáticos que se sitúan en transiciones discursivas mayores, como el turno, y aportan un significado estructural, propio del dominio textual, como el inicio o el fin de una unidad. 
En Cuenca y Crible (2019) se retoma la clasificación de Cuenca y Marín (2009) y se refina, además de aplicarla a un corpus de conversaciones y entrevistas en inglés, a partir del corpus de Crible (2017). Se considera que hay dos rasgos esenciales a la hora de diferenciar el grado de integración: el alcance de los marcadores (igual o diferente) y el significado (dos significados autónomos, dos combinados, uno solo). Según el primer rasgo, se diferencia la yuxtaposición (diferente alcance) y la combinación (igual alcance). En el caso de la combinación, el mayor o menor grado de integración tiene que ver con el significado y la función: los marcadores se suman (adición) si tienen funciones diferentes pero compatibles, mientras que forman un marcador compuesto (composición) si expresan una única función conjuntamente. Además, hay otros rasgos no esenciales pero importantes como la posición (inicial o interna), la categoría del marcador o las pausas (cfr. Lohmann y Koops, 2016).

La propuesta de Cuenca y Crible (2019), que explicaremos ulteriormente en el apartado siguiente y aplicaremos a conversaciones de Val.Es.Co, se resume en la figura 1.

\begin{tabular}{|l|l|l|}
\hline Type & Subtype & Distinctive features \\
\hline Juxtaposition & & Different scope, different functions \\
\hline Combination & Addition & $\begin{array}{l}\text {-Same scope, two different functions } \\
\text {-Same scope, two compatible but distinct functions }\end{array}$ \\
\hline & Composition & Same scope, one complex function \\
\hline
\end{tabular}

Figura 1. Tipos de secuencias de marcadores según el grado de integración (procedente de Cuenca y Crible 2019: tabla 7, p. 182)

En todas las contribuciones reseñadas se diferencian dos o tres grados de integración, pero los criterios y, sobre todo, las secuencias que entran en cada concepto son diferentes, especialmente en los casos de combinación, que son los más difíciles de delimitar y presentan límites difusos (cf. Cuenca y Crible, 2019: apartado 6).

Otro aspecto que ha llamado la atención de los investigadores es el orden de los marcadores. Lohmann y Koops (Koops y Lohmann, 2015; Lohmann y Koops, 2016) observan que ciertos marcadores tienden a aparecer juntos y que el orden es significativo. De su análisis de conversaciones telefónicas en inglés americano, concluyen que el orden de los elementos es muy sistemático: marcadores como las conjunciones, well o oh tienden a aparecer primero; marcadores como then, now, I mean tienden a aparecer en segundo lugar; en cambio, you know presenta ambas posiciones. Proponen la siguiente jerarquía de ordenación de los marcadores que analizan, siguiendo la lista de Schiffrin (1987):

(7) oh $>$ well $>$ and $>$ so $>$ or $>$ but $>$ because $>$ then $>$ you know $>$ now $>$ I mean (Lohmann and Koops, 2016: 433)

Desde una perspectiva similar, Tanghe (2018) analiza la tendencia a la concurrencia, el orden de los elementos y el grado de fijación de dos marcadores (uno de los cuales procede de un verbo de movimiento) usando un método cuantitativo que pone en 
relación la frecuencia individual de cada conector y la frecuencia absoluta de las secuencias. Igualmente, se ha observado una reversibilidad relativa de los elementos, de manera que algunos casos permiten el cambio de orden (bueno pues, pues bueno; oye pero, pero oye) pero otros no (bon ben vs. *ben bon) (cf. Waltereit, 2007; Lohmann y Koops, 2016; Pons, 2018).

Finalmente, algunos autores han observado que las secuencias pueden presentar procesos relacionados con la gramaticalización. Es el caso de Dostie (2013), que observa reducción fonológica de la unidad eh bien, que se convierte en eh ben, o de Crible (2015), que identifica una nueva ortografía de ciertas formas (ou sinon pasa a escribirse como aussi non) o cambios de posición, de inicial a final. Koops y Lohmann (2015) y Lohmann y Koops (2016) también relacionan la concurrencia de marcadores y su ordenación con procesos de gramaticalización; en concreto, consideran que la persistencia de las restricciones de orden de las formas de origen de los marcadores del discurso que se originan en el nivel oracional explica que unos precedan a otros.

Los fenómenos observados no dejan de ser facetas de una misma realidad compleja: la ordenación de los marcadores no es del todo independiente del grado de integración o de la categoría de los elementos adyacentes; por otro lado, la combinación de marcadores se puede relacionar con procesos de gramaticalización y pragmaticalización en curso o, simplemente, de rutinización que puede acabar con la lexicalización de formas inicialmente independientes.

\section{CLASIFICACIÓN DE LAS SECUENCIAS DE CONECTORES SEGÚN EL GRADO DE INTEGRACIÓN}

Como hemos dicho, partimos de la idea de que la yuxtaposición de dos o más conectores se da cuando tienen diferente alcance y, por lo tanto, distintas funciones. En cambio, hay combinación de conectores cuando tienen el mismo alcance y sus funciones se combinan de manera relativamente fuerte (adición), si los conectores suman sus funciones, o muy fuerte (composición), si la secuencia realiza una única función.

a) Yuxtaposición de conectores. Dos o más conectores están yuxtapuestos cuando tienen diferente alcance, es decir, introducen unidades sintácticas diferentes: una unidad mayor y una unidad menor, que forma parte de la primera.

(8) J: nada/ resulta quee estaba lloviendo/ ¿no?/ fue el fin de semana pasao/ yy mi hermana se iba de boda/ yy yo no me acordaba/ [y [como yo me voy por ahí los sábados por la tarde con el coche]/ y estamos en el coche y eso/ pues le dije que claro/ que no tenía coche] [y [como estaba lloviendo por ahí/] no era plan de irse de pafs $\downarrow$ ni nada de eso/ [aunque [cuando llegué] ya no llovía $\uparrow$ ] pero ya puestos $\uparrow$ digo $\rightarrow$ pues vamos a su casa y ya está $\uparrow \S](14,130)$

En (8) ilustramos la concurrencia de dos conjunciones en tres casos: la primera conjunción tiene un alcance mayor que la segunda, y sus funciones y significados aparecen claramente diferenciados $(y$ como = adición + causa; aunque cuando = concesión + tiempo). 
Aunque la configuración más frecuente de la yuxtaposición de conectores es la de dos conjunciones consecutivas, también es posible encontrar otras configuraciones, que incluyen conectores parentéticos (9) o pragmáticos (10).

(9) $P$ : entonces cuando le metíaa $\uparrow$ el sabe que aqueel $\uparrow(15,34)$

(10) A: bueno/ si quieres dejamos el tema $(())(1,495)$

Los casos más frecuentes de yuxtaposición que aparecen en el corpus son: $y$ que, $y$ como, pero que, pero como, pero si, que bueno y que si.

b) Adición de conectores. Dos conectores se suman cuando tienen el mismo alcance, pero presentan significados distintos aunque compatibles; el segundo conector precisa o refuerza el significado del primero. La configuración típica de estas secuencias es la que incluye una conjunción seguida de un conector parentético, como en (11) y (12).

(11) A: es más divertido $\rightarrow /$ no sé/ yo qué sé/ [y además/ se montan muchas verbenas $\rightarrow$ al aire libre=] (1, 391)

(12) P: pero de todas formas eso// ee// lo- lo que estamos de carrera/ problemas con los neumáticos $\uparrow$ y que yaa $\uparrow$ estaa- estás cansao§ $(15,106)$

Con todo, en algunos casos podemos encontrar otras configuraciones, como en (13), donde el conector pragmático bueno va seguido de un conector parentético (en realidad), que indican reorientación y contraste (o, si se quiere, contraste en el dominio textual o estructural y en el ideacional o proposicional).

(13) B: la lengua de los gitanos $/ / /(1,11) \mathrm{hmm} / /$ bueno $\downarrow$ en realidad el tratamiento lexicográfico que se le daa a los que se le daa a los gitanismos een en los diccionarios actuales. $(42,72)$

Los casos más frecuentes de adición que aparecen en el corpus son: y además, $y$ encima, $y$ entonces (equivalente a $y$, por lo tanto) y que por cierto.

c) Conectores compuestos. Dos conectores forman una unidad compleja cuando tienen el mismo alcance y expresan un único significado.

(14) A: [no gritar pero $\rightarrow$ ] sí sí sí $\downarrow /$ pero- no GRITAR/ pero ¿por qué no se tiene que gritar $\uparrow$ ?/ y yo si quiero gritar ¿qué pasa?/ y ellos me decían $\uparrow$ no no/ no hay que gritar pues eso $\downarrow$ por lo que ya les había dicho yo $\downarrow /$ a lo mejor había un señor leyendo $\uparrow$ y le molestábamos $\uparrow$ o estaba escuchando la radio $\uparrow$ o quería hablar con alguien $\uparrow \mathbf{y}$ entonces $\uparrow$ pues los gritos le molestaban/ y entonces $\uparrow$ yo decía no no pues a mí me apetece gritar// (RISAS) [y entonces les decía $\rightarrow$ ] $(1,247)$

En (14), observamos tres usos de y entonces que marcan fronteras en la narración y, en los dos últimos casos, introducción de discurso directo del hablante. La secuencia adopta el significado estructural de continuidad, que contienen separadamente los dos elementos, pero que se integra en casos como estos. A la unidad semántica, se suma la prosódica y la estructural: no es posible insertar una pausa intermedia estructural entre los dos conectores, existe un tonema ascendente después del segundo conector (excepto en el último caso, que, de hecho, resulta ambiguo pues admite una lectura temporal de entonces) y no admite sustitución del segundo elemento (sustitución que admitiría si entonces tuviera valor consecutivo, por ejemplo por en consecuencia, caso en el que se trataría de una adición de conectores, no de un conector compuesto). 
Desarrollaremos el análisis este tipo de secuencia en el siguiente apartado.

\section{CONECTORES COMPUESTOS: CONCEPTO Y LÍMITES}

Los conectores compuestos incluyen dos conectores que pueden actuar autónomamente pero que, en combinación, introducen una misma unidad del discurso y realizan una única función discursiva. En palabras de González (2004: 297), "[their] combinatory functions result in a change of attentional state of the speaker, or shift in cognitive frame, and/or a remarkable emphasis on the illocutionary point of the segment".

Los conectores compuestos en español suelen corresponder a una conjunción seguida de un conector parentético o pragmático. En el primer grupo encontramos las secuencias $y$ entonces, y luego y pues entonces, que tienen funciones estructurales relacionadas con la distribución de la información, la enumeración y la continuidad. En el apartado anterior, analizamos tres casos de $y$ entonces. Veamos un ejemplo de $y$ luego (15):

(15) A: hayy $\uparrow$ - hay cuatro ramas/ que son/ la de humanas $\uparrow /$ que es todo lo de historia y todo esto $\uparrow$ filología $\downarrow$ que son lenguas $\rightarrow \S$

B: § lenguas $\downarrow[$ sí]

A: $[(())]$ lengua española $\uparrow$ como lo tuyo// lengua española/ inglés $\uparrow /$ bueno $\downarrow$ francés y todo esto $\uparrow /$ luego estáa ciencias $\uparrow / /$ que es cosa ya dee mate[máticas y física $\uparrow]$

B: [matemáticas $\rightarrow$ ] y todo esto

A: y preescolar $\uparrow$ que ya es $\uparrow$ pues paraa [pues primero y segundo de preescolar $\downarrow$ ]

B: [para eso $\uparrow$ también $\uparrow$ otra especialidad]

A: y luego aparte hay una que en Godella no- no la dan个/ que es e- educación especial ¿no?/ para los niños con deficiencias $\rightarrow \S$

B: $\S$ o sea tú fuiste a Godella

A: yo fui a Godella

B: y luego aquí en Valencia $\uparrow$ hay otra aquí en Jacinto Bena- ¡bueno! al final de [Jacinto Benavente $\uparrow]$ $(1,29-38)$

En (15), el hablante A está enumerando las diferentes especialidades de Magisterio: introduce las tres primeras (humanas, ciencias y preescolar) y añade la cuarta (educación especial) separándola con una secuencia de marcadores ( $y$ luego aparte), porque, a diferencia de las tres anteriores, no se imparte en el centro de Godella. El hablante $B$, en la última intervención, reorienta el discurso con la secuencia y luego que introduce una nueva información: hay otro centro, el de la Universitat de València. En estos casos, luego no significa en un momento posterior ni tiene una función por sí mismo, sino que es la unidad compleja y luego la que estructura el discurso. En la secuencia de tres elementos, y luego aparte, el tercero se une aditivamente (podríamos decir y luego además).

La secuencia pues entonces (16) parece tener propiedades similares a y entonces o $y$ luego.

(16) (16) A: = y lo sacas/ peroo si es cosa ya dee/ unn proyecto $\uparrow /$ que en ese tribunal le gusta o no le gusta $\uparrow$ pues entonces $\uparrow / /$ te lo aprueban o no te lo aprueban dependiendo dee§ $(1,5)$

En la mayor parte de estos ejemplos, el tonema final suele ser ascendente y no hay pausa intermedia entre los conectores, ni sería natural introducirla, a diferencia de lo 
que sucede en los casos de adición, donde es posible hacer una pausa o inflexión descendente intermedia y final.

Estas configuraciones resultan a veces difíciles de diferenciar de secuencias formalmente iguales, debido a la polifuncionalidad del segundo elemento. Luego y entonces pueden ser adverbios de tiempo, conectores parentéticos de consecuencia o elementos de un conector compuesto. A pesar de la relación diacrónica y sincrónica de las tres funciones, el significado (y las paráfrasis que implican) ayuda a diferenciarlos.

(17) J: § no/ y luego se lo dije a Migue $\downarrow$ y dice $\uparrow$ juy! ¿qué tu hermano no sube o qué? $\uparrow$ dice $\uparrow$ ahora $\downarrow$ hoy se ha ido que está preparando una acampada $\downarrow$ o no sé qué/ o sea que se habrá ido todas las pascuas de acampada $(14,104)$

(18) $A$ : = o sea es una concentración en ti mismo/ y entonces no abarcaas/ al resto de la gente $(1,173)$

Así, cuando se trata de adverbios, como en (17), luego equivale a después y entonces equivale a en aquel momento. En casos como (18) entonces tiene valor consecutivo y equivale a en consecuencia. En el primer caso, no existe secuencia de conectores, pues el segundo elemento es un adverbio de tiempo; en el segundo caso, existe adición de conectores. Cuando estas sustituciones no son posibles, nos encontramos ante casos de conectores compuestos.

El segundo grupo de conectores compuestos identificados incluye una conjunción básica seguida de un conector pragmático (bueno, mira, vamos): y bueno, pero bueno, pero mira, pero vamos, pues bueno, pues mira.

(19) B: pues que me caí ((puees)) bebí un poquito de rusc $\rightarrow / / /(1,71)$ de rusco (RISAS) me bajó la tensión $\rightarrow$ / y me caí rodando / [me encontraron (( ))] (( )) ((en el suelo)) todos mis colegas buscándome* (RISAS) y bueno así pasó $(42,18)$

(20) D: pero claro el Aspar también era un desmayao y ha tenido/ pero bueno el Aspar lleva muchos añoos $\uparrow /$ ha sido $\uparrow$ cinco veces campeón del mundo/ yy tiene eel- el re- respaldadero de la Generalitat Valenciana que le ha apoyao/ y como está ganando resultaos $\uparrow$ pero $\uparrow$ es por el esponsor/// $(15,167)$

(21) A: yaa/ ya lo sé $\downarrow$ pero mira/ eso es lo que hay/ ¿qué quieres que te diga? [yo entonces $\uparrow$ claro $\downarrow=$ ] (1, 15)

(22) B: no/ además/ un buen director $\uparrow$ yo de música no entiendo mucho/ pero vamos/ por lo menos tiene que darle un poquitín dee§ $(1,497)$

(23) B: ((en)) lengua y litertura pero jjolines! / ¡son ((unoos)) unos bestias! / jsetenta y dos temas! y ahíi quee // pues bueno yo no tengo ni idea de oposiciones ¿no? eso es lo que han conta(d)o loos los colegas que se han presentao ¿no? así ¿no? con bolas / te salen tres bolas $\uparrow$ // como si fuera el bingo $\uparrow$ $\S(42,474)$

(24) A: pues mira $\uparrow /$ moverme $\uparrow$ me he movido poco// los $\uparrow-/$ a lo máximo de lejos que he llegado ha sido a Lourdes $(1,551)$

Se trata de conectores complejos con un valor estructural-modal que contribuyen a pautar el discurso integrando el valor de la conjunción y el del conector pragmático. La conjunción no se interpreta en el dominio del contenido como aditiva, contrastiva o consecutiva, sino en el dominio estructural como iniciativa (pues), continuativa $(y)$ o reorientativa (pero); por su lado, el conector pragmático (bueno o una forma derivada 
de un verbo de percepción o de movimiento) ${ }^{5}$ añade un matiz atenuativo a la unidad compleja.

El valor modal resulta más marcado, en detrimento del valor conectivo, en estructuras como las de (25) i (26), en las que el segundo elemento de la conexión está implícito o incluso resulta innecesario. ${ }^{6}$

(25) B: § porque las verbenas $\uparrow$ a mí también me gustan bastante// pero mira $\rightarrow(1,390)$

(26) $B:=$ un poco fuerte/ ¿eh? pero vamos $\rightarrow(1,464)$

El concepto de conector compuesto es relativamente fácil de delimitar intuitivamente, pero no a partir de pruebas que los distingan claramente de la simple adición de conectores (o de ciertos efectos colocacionales), por un lado, y de los conectores lexicalizados pluriverbales, de otra. Así, es fácil ver la diferencia de integración entre pues bueno, pero bueno o pues bien, por un lado, y bueno pues, bueno pero o bien pues, por otro, como indican Pons (2008: 157-158) o Tanghe (2018: 800) y se puede observar en los ejemplos siguientes:

(27) A: [bueno pero] porque estás haciendo el proyecto de [investigación] $(42,41)$

(28) C: [pero bueno así] se exhibe [y luego entra gratis a la discoteca] $(42,284)$

En el primer caso (25), los dos marcadores tienen usos diferenciables: bueno equivale a de acuerdo y pero introduce un contraste; en palabras de Pons, entre los dos elementos "existe una frontera de unidad (subacto o acto), de modo que la supuesta combinación de conectores no es tal, sino adyacencia de dos funciones discursivas, de las que la primera tiene ámbito sobre la segunda" (Pons 2008: 157). En el segundo caso (26), los dos elementos forman una única unidad: bueno no admite paráfrasis o conmutación ( ${ }^{*}$ pero de acuerdo) y actúa como una unidad conectiva compleja con la que el hablante indica un contraste atenuado (cfr. también Pons 2018).

Por otro lado, cabe diferenciar los conectores compuestos de unidades lexicalizadas como así pues, que se entienden como unidades conectivas "de diccionario", totalmente equiparables a otras unidades pluriverbales, como por lo tanto o en consecuencia, que actúan como conectores parentéticos y expresan valores proposicionales 0 estructurales, en este caso de consecuencia o continuidad discursiva.

\section{CONCLUSIONES}

En este trabajo, nos hemos propuesto un objetivo relativamente modesto: analizar los tipos de secuencias de conectores a partir de un corpus reducido compuesto por cuatro conversaciones del corpus Val.Es.Co. Para ello, hemos recurrido a la propuesta que se

\footnotetext{
${ }^{5}$ Sobre bueno en combinaciones, véase González (2001, 2004), Pons (2008: apartado 5) y Cuenca y Marín (2009: apartado 3); en cuanto a verbos de movimiento combinados, véase Tanghe (2018), quien ofrece un análisis cuantitativo para abordar el grado de fijación sintagmática de estas unidades combinadas entre sí, con las conjunciones y, pero, porque, pues y con otros marcadores como bueno, hombre, a ver y vale. Cabe notar, sin embargo, que algunos de los casos analizados en la bibliografía no son secuencias de conectores en sentido estricto, sino de marcadores discursivos o pragmáticos, en sentido amplio.

${ }^{6}$ Existen otros casos de construcciones "suspendidas", como las que incluyen al contrario en español y formes similares en otras lenguas (véase Cuenca y Visconti, 2017; Cuenca y Estellés, en prensa).
} 
presenta en Cuenca y Crible (2019) aplicada a un corpus de inglés oral y que ya se había aplicado, de manera más global, a narraciones orales en catalán y español (Cuenca y Marín 2009).

Hemos visto que la mayoría de secuencias contienen dos conectores, si bien existen secuencias de tres y cuatro conectores. El primer conector suele ser una conjunción general (sobre todo y y pero), que puede tener uso en el nivel oracional como elemento introductor de una oración compuesta pero también en el nivel textual, asociado con el dominio textual y las funciones estructurales. También tiene una frecuencia alta en primer lugar el conector pragmático bueno, que, a diferencia de las conjunciones, se pueden encontrar frecuentemente en segundo lugar (17 casos, frente a 13 casos). El segundo conector de la secuencia es típicamente un conector parentético (entonces, luego, además) o pragmático (bueno, vamos (a ver)), aunque algunas conjunciones subordinadas también se yuxtaponen a otros conectores (como, que, si).

Los resultados obtenidos para la conversación en español son parecidos a los que ofrece el corpus inglés analizado en Cuenca y Crible (2019): los conectores más frecuentes en posición inicial son and, but, so, well y because; los más recuentes en segunda posición son if, so, then, I mean. Igualmente son comunes las secuencias más frecuentes: $y$ entonces ( 21 casos) y luego (18 casos), en español, y and so (15 casos) $\mathrm{y}$ and then (13 casos) en inglés.

Hemos ilustrado casos de yuxtaposición de conectores, que son fácilmente identificables, en general, puesto que el alcance de los mismos es distinto, y casos de combinación, en los que los conectores implicados tienen alcance sobre la misma unidad. La combinación puede implicar una integración menor (adición de conectores) o mayor (conectores compuestos). El grado de integración afecta a la autonomía o la unidad de función y significado. Ciertamente, la unidad de significado resulta, a veces, difícil de determinar por lo que los límites entre la adición y la composición pueden resultar difusos. Con todo, las pruebas de paráfrasis o conmutación y la presencia o ausencia de pausas o inflexiones pueden ayudar a diferenciar el mayor o menor grado de integración. En el caso de los conectores compuestos no se admite la conmutación de uno de los elementos por otro conector ni tampoco la inserción de pausa intermedia sin cambio de significado, a diferencia de lo que se observa en casos de adición. Además, la función de un conector compuesto no se sitúa en el dominio proposicional sino en el dominio estructural o estructural-modal.

Cuantitativamente, los ejemplos identificados en conversaciones en español corresponden mayoritariamente a casos de yuxtaposición (73 ejemplos), seguidos por los de composición (59 casos) y los de adición (36 casos). Estos resultados contrastan parcialmente con los del inglés: la yuxtaposición es también la relación más frecuente (50 casos), pero la adición (34 casos) supera a la composición de conectores (25 casos). Esta diferencia puede deberse a una preferencia de la retórica del inglés por el uso de marcas estructurales en comparación con el español (y probablemente otras lenguas románticas) en las que el marcaje estructural-modal es más frecuente.

El análisis que hemos presentado aquí, aunque preliminar, selectivo en cuanto a los rasgos que se describen y limitado a un corpus relativamente pequeño, pretende ser un 
banco de pruebas de un análisis más extenso que podría hacerse en colaboración con el grupo Val.Es.Co. De hecho, en el marco de este grupo de investigación ya se han hecho algunas aportaciones al tema, especialmente de la mano de Salvador Pons $(2008,2018)$, que pueden servir de base para una ampliación de este estudio tanto desde el punto de vista descriptivo como de contraste lingüístico.

\section{BIBLIOGRAFÍA}

CRIBLE, Ludivine (2015): «Grammaticalisation du marqueur discursif complexe ou sinon dans le corpus de SMS belge: spécificités sémantiques, graphiques et diatopiques», Le Discours et la Langue 7(1): 181-200.

CRIBLE, Ludivine (2017): «Discourse markers and (dis)fluencies in English and French: Variation and combination in the DisFrEn corpus», International Journal of Corpus Linguistics 22 (2), 242-269.

CRIBLE, Ludivine (2018): Discourse Markers and (Dis)fluency. Forms and Functions across Languages and Registers, Amsterdam, John Benjamins.

CuENCA, Maria Josep (2001): «Los conectores parentéticos como categoría gramatical», Lingüística Española Actual, XXIII 2, 211-235.

CuENCA, Maria Josep (2006): La connexió $i$ els connectors. Perspectiva oracional i textual, Eumo, Vic.

CuENCA, Maria Josep (2013): «The fuzzy boundaries between modal and discourse marking», en Liesbeth Degand, Bert Cornillie y Paola Pietrandrea (eds.), Discourse Markers and Modal Particles: Description and Categorization. Amsterdam, John Benjamins, 191-216.

CuencA, Maria Josep y Ludivine CRIBLE (2019): «Co-occurrence of discourse markers in English: from juxtaposition to composition», Journal of Pragmatics, 140, 171-184.

CuenCA, Maria Josep y Maria Estellés (en prensa): "Los marcadores contrastivos antes bien, antes al contrario y al contrario en español actual», en Catalina Fuentes Rodríguez, Antonio Messias Nogueira da Silva y Manuel Martí, Marcadores discursivos en español y portugués, UAH.

CUENCA, Maria Josep y Maria Josep MARín (2009): «Co-occurrence of discourse markers in Catalan and Spanish oral narrative», Journal of Pragmatics, 41, 899-914.

CUENCA, Maria Josep y Jacqueline VISCONTI (2017): «De la precedencia temporal al contraste: el marcador del discurso ans en catalán y anzi en italiano», en Mercedes Banegas Saorín y Véronique Lagae (eds.). Pragmalingüística, Monográfico 1, Marqueurs discursifs des langues romanes: approches croisées, 108-130.

DostIE, Gaëtane (2013): «Les associations de marqueurs discursifs - De la cooccurrence libre à la collocation», Linguistik Online 62 (5).

FRASER, Bruce (2013): «Combinations of contrastive discourse markers in English», International Review of Pragmatics, 5, 318340.

FRASER, Bruce (2015): "The combining of discourse markers - A beginning», Journal of Pragmatics, 86, 48-53.

GonZÁLEZ CONDOM, Montserrat (2001): «Els marcadors pragmàtics compostos en el relat oral anglès i català», Caplletra, 30, 73-93.

GonZÁlez Condom, Montserrat (2004): Pragmatic Markers in Oral Narrative: the Case of English and Catalan, Amsterdam, John Benjamins.

Hansen, Maj-Britt Mosegaard (1998): The Function of Discourse Particles. A Study with Special Reference to Spoken Standard French, Amsterdam, John Benjamins.

Koops, Christian y Arne LoHMANN (2015): «A quantitative approach to the grammaticalization of discourse markers», International Journal of Corpus Linguistics 20 (2), 232-259.

LoHmAnN, Arne y Christian Koops (2016): «Aspects of discourse marker sequencing: Empirical challenges and theoretical implications», en Kaltenböck, Evelien Gunther, Keizer y Arne Lohmann (Eds.), Outside the Clause: Form and Function of Extra-clausal Constituents, Amsterdam, John Benjamins, 417-446.

LUSCHER, Jean-Marc (1993): «La marque de connexion complexe», Cahiers de Linguistique Française, 14, 173-188.

PoNs, Salvador (2008): "La combinación de marcadores del discurso en la conversación coloquial: interacciones entre posición y función», Estudos Linguísticos/Linguistic Studies, 2. Lisboa, Edições Colibri/CLUNL, 141-159.

PoNs, Salvador (2018): «The combination of discourse markers in spontaneous conversations: Keys to undo a gordian knot», Revue Romane, 53 (1), 121-158. 
REDEKER, Gisela (1990): «Ideational and pragmatic markers of discourse structure», Journal of Pragmatics 14 (3), 367-81.

ScHIFFRIN, Deborah (1987): Discourse markers. Cambridge (Mass.): Cambridge University Press.

TANGHE, Sanne (2018): «Combinaciones de marcadores del discurso en el lenguaje hablado: un estudio empírico de anda, vamos, vaya y venga», Rilce, 34(2), 792-819.
WALTEREIT, Richard (2007): «À propos de la genèse diachronique des combinaisons de marqueurs. L'exemple de bon ben et enfin bref», Langue française, 157 (2), 94-109.

\section{Corpus}

Cabedo, Adrián y Salvador Pons (eds.): Corpus Val.Es.Co 2.0. Consultado online en http://www.valesco.es. 